\title{
Centres d'urgence hospitaliers: Recommandations structurelles et organisation- nelles en matière d'assurance qualité
}

Groupe de travail de la Communauté d'intérêts directeurs médicaux des urgences *

Deutsch erschienen

in Nr. 32-33/2005
Les synonymes suivants sont utilisés pour les «services d'urgence»:

- Centres d'urgence (f)

- Pronto Soccorso (i)

- Notfallstation (d)

- Notfallaufname (d)

- Notfallzentrum (d)

\section{Antécédents}

La Communauté d'intérêts directeurs médicaux des urgences est une association récente qui depuis janvier 2002 est placée sous le parrainage de la SSMUS (Société Suisse de Médecine d'Urgence et de Sauvetage). Elle se destine à améliorer la qualité fonctionnelle des centres d'urgence par l'entremise d'un certain nombre de critères structurels et organisationnels ayant valeur de standards. Son second but est de permettre les échanges scientifiques et d'idées entre les centres.

Une première version de ce guide a fait l'objet en avril 2003 d'une large consultation auprès des Services cantonaux de santé, des directions d'hôpitaux, d'organisations et de collègues. Les nombreuses remarques, suggestions et critiques (>100) ont permis la finalisation de ce document.

\section{Introduction}

La médecine d'urgence ne constitue pas encore en Suisse une spécialité médicale à part entière. Elle n'est pas reconnue en tant que telle par la $\mathrm{FMH}$, si ce n'est sous forme d'un certificat de capacité de médecin d'urgence, dont les caractéristiques ont été fixées par la SSMUS (Société Suisse de Médecine d'Urgence et Sauvetage) et concernent exclusivement la médecine d'urgence préhospitalière.

Malgré ceci, l'ensemble des hôpitaux suisses assumant des urgences a vu au cours des dernières années une augmentation importante de leur activité, observée de manière identique dans tous les pays occidentaux, et imposant une adaptation des structures médicales, infirmières et d'organisation, voire également architecturales, sans qu'un référentiel de qualité concernant ces points ne soit disponible en Suisse. Dès lors, ce sont des aménagements locaux qui ont été faits, dont le contenu a bien plus pris en compte des avis personnels et préservé des structures historiques locales que remis en question la structure fondamentale des services d'urgence et redéfini leurs missions. On trouve donc à ce jour une myriade de structures de direction, d'organisation médicale ou infirmière, ou encore d'organisation des flux de patients, des ressources en locaux ou en services de soutien.

Malgré cette disparité organisationnelle, l'ensemble de ces centres d'urgence est exposée à un certain nombre de problèmes communs, dont, pour exemple, on peut citer la direction du Centre, l'organisation médicale, la collaboration interdisciplinaire, la répartition des ressources etc.

Les auteurs de ce guide se sont réunis pour proposer un certain nombre de critères de qualité quand à la structure des centres d'urgence suisses. Ils ont reçu pour ce faire mandat de la Société suisse de médecine d'urgence et de sauvetage (SSMUS), qui parraine ce travail. Outre l'utilisation de ce guide à fin de référentiel de structure pour la modernisation ou la construction nouvelle de centres d'urgence, il se veut également être un outil d'évaluation permettant l'étude et la comparaison des centres d'urgence actuels.

Les auteurs ne considèrent pas les critères mentionnés ci-dessous comme des conditions actuelles, mais surtout comme des buts à atteindre dans les années prochaines.

\section{Méthode}

Le groupe de travail a utilisé les référentiels structurels existant dans la littérature [1-10] et les a adaptés à la situation suisse plutôt que de développer de novo un référentiel entièrement nou- 
veau. Dans ce travail d'adaptation, le groupe s'est efforcé de prendre en compte le contexte sanitaire suisse, en particulier la dimension moyenne voire modeste des centres d'urgence helvétiques (voir ci-dessous), le contexte d'une organisation cantonale des soins, l'existence de centres de référence de divers types (centres cantonaux et centres universitaires). En aucune manière ce référentiel ne peut s'appliquer tel quel à toutes les structures hospitalières suisses; il doit plutôt être considéré comme une liste de propositions idéales («critères d'optimalisation») à adapter selon le contexte local et régional concerné.

Par contre, le groupe de travail a estimé souhaitable le maintien d'un concept fondamental général de multidisciplinaritépour les médecins actifs au sein des centres d'urgence.

Les auteurs de ces recommandations sont de l'avis que toute modification structurelle ou organisationnelle d'un centre d'urgence doit primairement être justifiée par l'intérêt des patients. Les structures historiques des services cliniques hospitaliers ne peuvent en aucun cas prévaloir sur cette priorité. $\mathrm{Au} v \mathrm{vu}$ des différences importantes de taille des centres d'urgence en Suisse, les auteurs de ces recommandations les ont classés en trois groupes.
Cette méthode de classification a été un des points les plus discutés lors de la consultation de 2003. Elle ne vise ici qu'à la description pratique des aspects structurels et organisationnels.

En aucun cas, cette classification n'a pour buts ou ne devrait être utilisée pour:

- établir des différences de tarification des prestations;

- constituer un guide pour l'orientation des patients dans la chaîne des urgences;

- accréditer les centres d'urgence dans leur mission de formation.

Le groupe de travail a pris connaissance du projet de la Plate-forme de sauvetage de la FMH, dont l'intention est de créer une catégorisation des centres d'urgence dans le but d'améliorer l'orientation des patients selon leur gravité.

En accord avec la Plate-forme FMH, il a été décidé de ne pas associer les deux classifications pour le moment, car leurs buts sont totalement différents.

Si les travaux de la Plate-forme FMH aboutissent et sera acceptés et appliqués dans l'ensemble du pays, les présentes recommandations seront révisées et adaptées à la classification FMH.

Tableau 1

Classification des centres d'urgences suisses dans trois groupes. Voir les remarques ci-dessous!

\begin{tabular}{|c|c|c|}
\hline Groupe & Description & Consultations \\
\hline III & $\begin{array}{l}\text { Centre d’urgence d'un hôpital de référence: fonctionne comme hôpital tertiaire. } \\
\text { Majorité des disciplines spécialisées disponibles } 24 / 24 \text { h }\end{array}$ & $>15000 / a n$ \\
\hline \multirow[t]{3}{*}{ II } & $\begin{array}{l}\text { Centre d'urgence d'un hôpital cantonal ou régional important disposant } \\
\text { de moyens médicaux conséquents }\end{array}$ & 7000-20000/an \\
\hline & $\begin{array}{l}\text { Disciplines de base disponibles } 24 / 24 \text { h (= chirurgie / orthopédie-traumatologie / } \\
\text { médecine interne / gynécologie-obstétrique). }\end{array}$ & \\
\hline & $\begin{array}{l}\text { Le transfert secondaire de patients vers un hôpital de référence est organisé } \\
\text { et fonctionnel. }\end{array}$ & \\
\hline \multirow[t]{3}{*}{ I } & $\begin{array}{l}\text { Centre d'urgence d'un petit hôpital périphérique: hôpital cantonal ou régional } \\
\text { de petite taille, assumant l'accueil des urgences } 24 / 24 \text { h, avec une capacité } \\
\text { médicale limitée. }\end{array}$ & $<10000 / a n$ \\
\hline & $\begin{array}{l}\text { Les mêmes critères sont applicables (avec certaines limitations) pour l'accueil } \\
\text { des urgences dans les permanences. }\end{array}$ & \\
\hline & $\begin{array}{l}\text { Le transfert secondaire de patients vers un hôpital de référence est organisé } \\
\text { et fonctionnel. }\end{array}$ & \\
\hline \multicolumn{3}{|c|}{ Remarques } \\
\hline \multicolumn{3}{|c|}{$\begin{array}{l}\text { Le groupe de travail a choisi la dénomination des groupes dans cet ordre pour respecter les habitudes de la litté- } \\
\text { rature, où en parle de soins primaires = soins de base, et respectivement de soins tertiaires = soins maximaux. }\end{array}$} \\
\hline \multicolumn{3}{|c|}{$\begin{array}{l}\text { Le nombre de consultations ne constitue pas un critère absolu. La classification doit plutôt considérer les autres } \\
\text { caractéristiques de l'hôpital pour l'attribution des urgences en groupe III ou II. }\end{array}$} \\
\hline \multicolumn{3}{|c|}{$\begin{array}{l}\text { Pour les centres d'urgence prenant en charge moins de } 10000 \text { patients par année, seuls des critères minimaux } \\
\text { ont été établis. Il est en effet difficile d'établir des critères optimaux pour ces centres d'urgence, car ils dépendent } \\
\text { trop de la situation géographique et de la structure hospitalière locale. Une adaptation aux critères de degré II est } \\
\text { conseillée de cas en cas. }\end{array}$} \\
\hline
\end{tabular}




\begin{tabular}{|c|c|c|c|c|c|c|c|c|c|c|}
\hline & 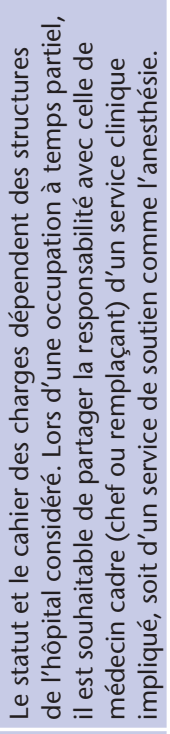 & 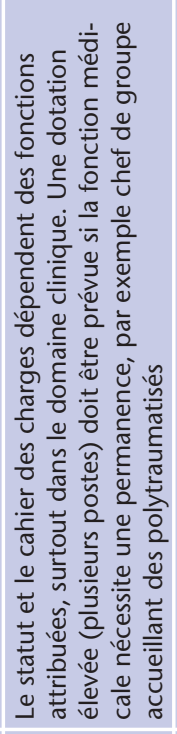 & 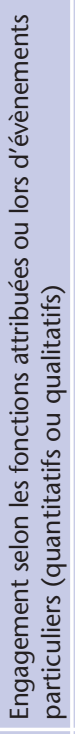 & 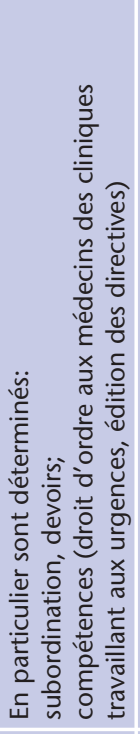 & 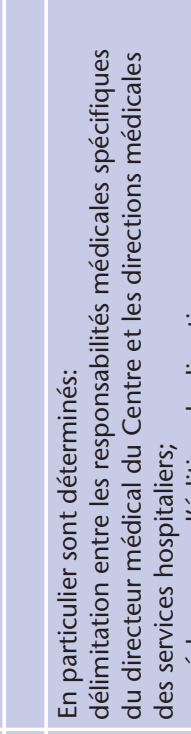 & 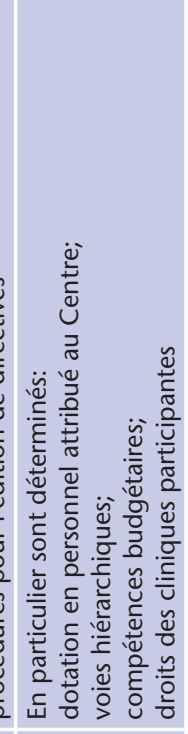 & 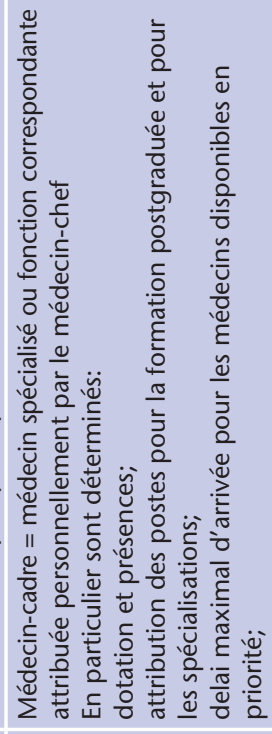 & 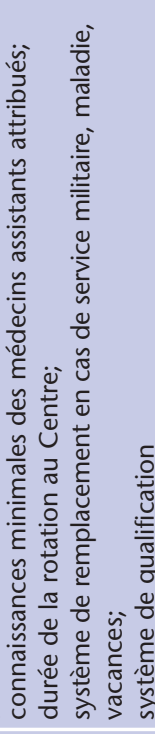 & 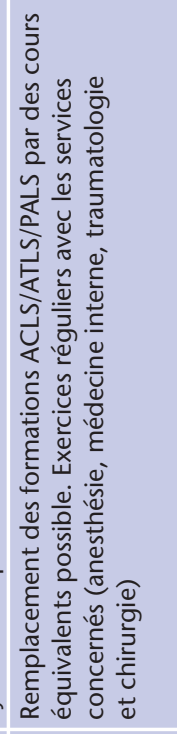 & 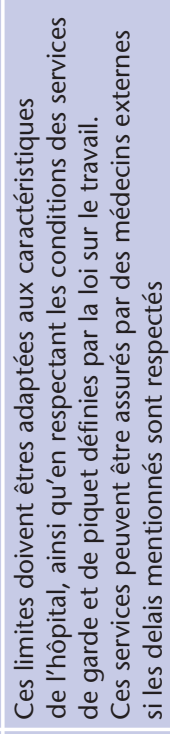 \\
\hline $\begin{array}{l}\overline{0} \\
\overline{0} \\
\overline{0} \\
\text { vo }\end{array}$ & 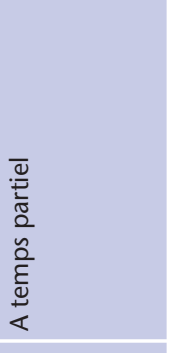 & 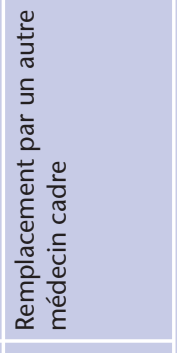 & \multirow{2}{*}{ 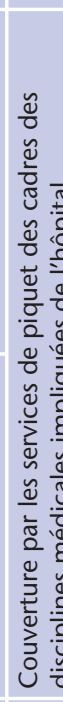 } & \multirow{3}{*}{ 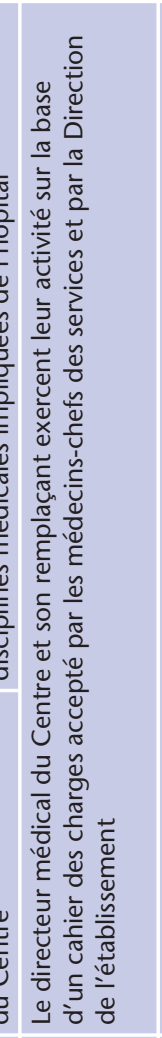 } & \multirow{3}{*}{ 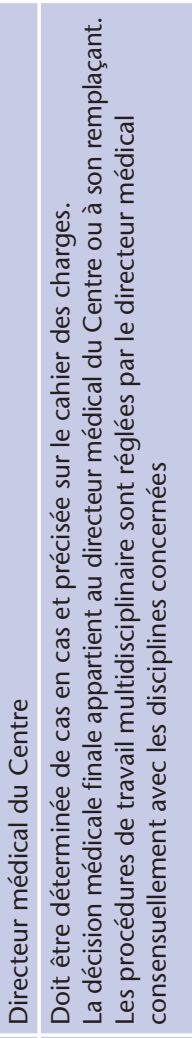 } & 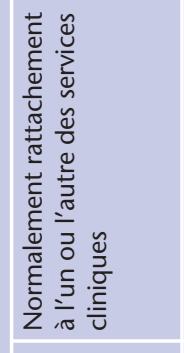 & 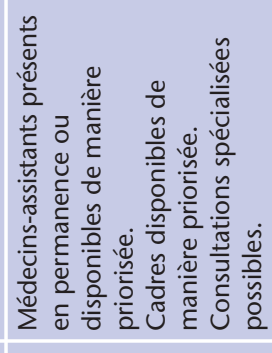 & & 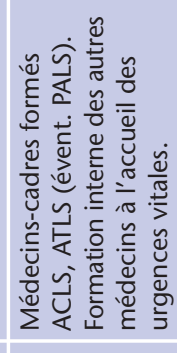 & \multirow{3}{*}{ 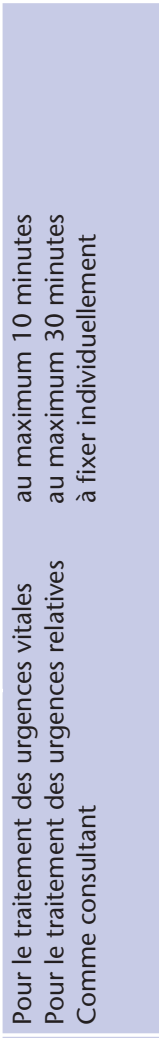 } \\
\hline & 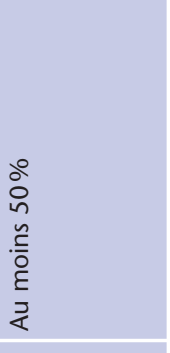 & 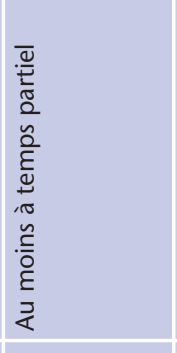 & & & & 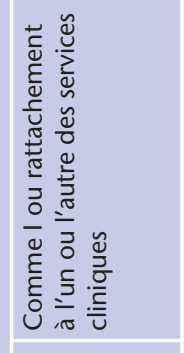 & 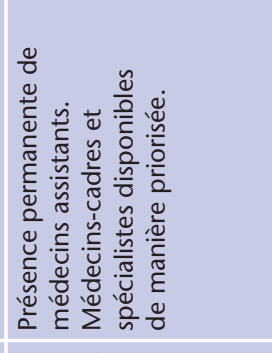 & & 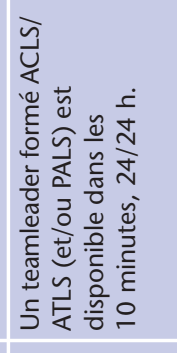 & \\
\hline & 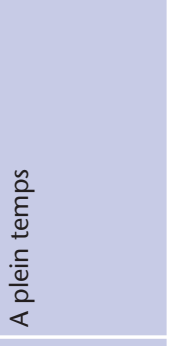 & 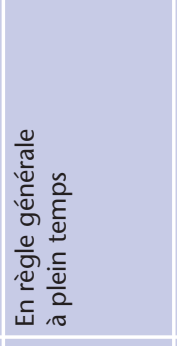 & 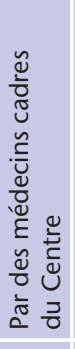 & & & 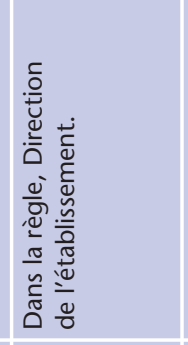 & 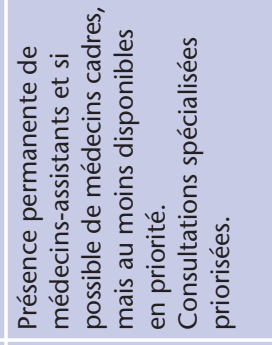 & & 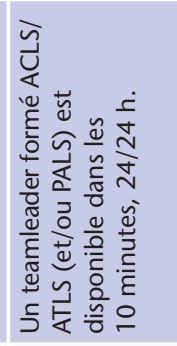 & \\
\hline 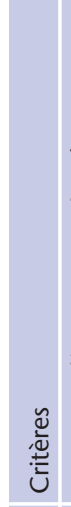 & 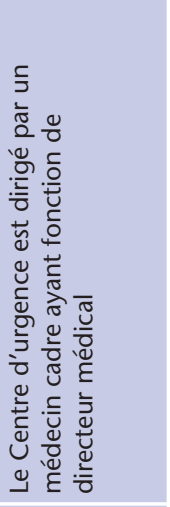 & 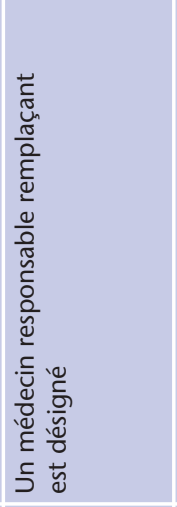 & 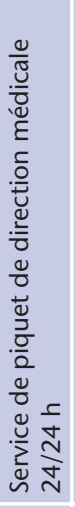 & 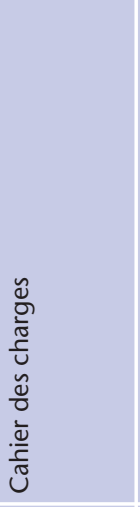 & 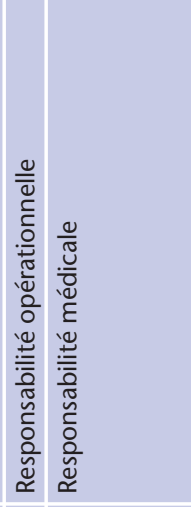 & 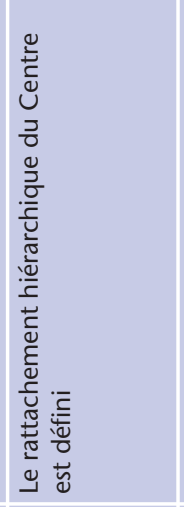 & 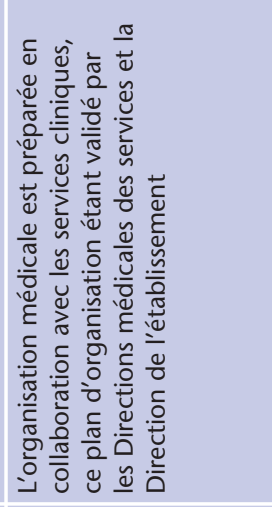 & & 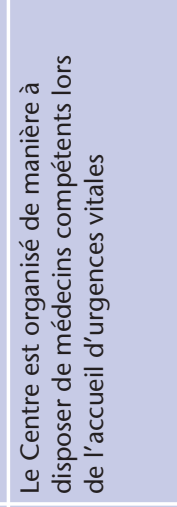 & 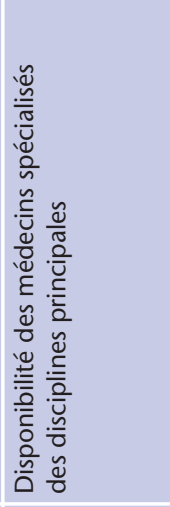 \\
\hline & $\varangle$ & $\infty$ & $u$ & D & ш ᄂ & u & I & & - & - \\
\hline
\end{tabular}




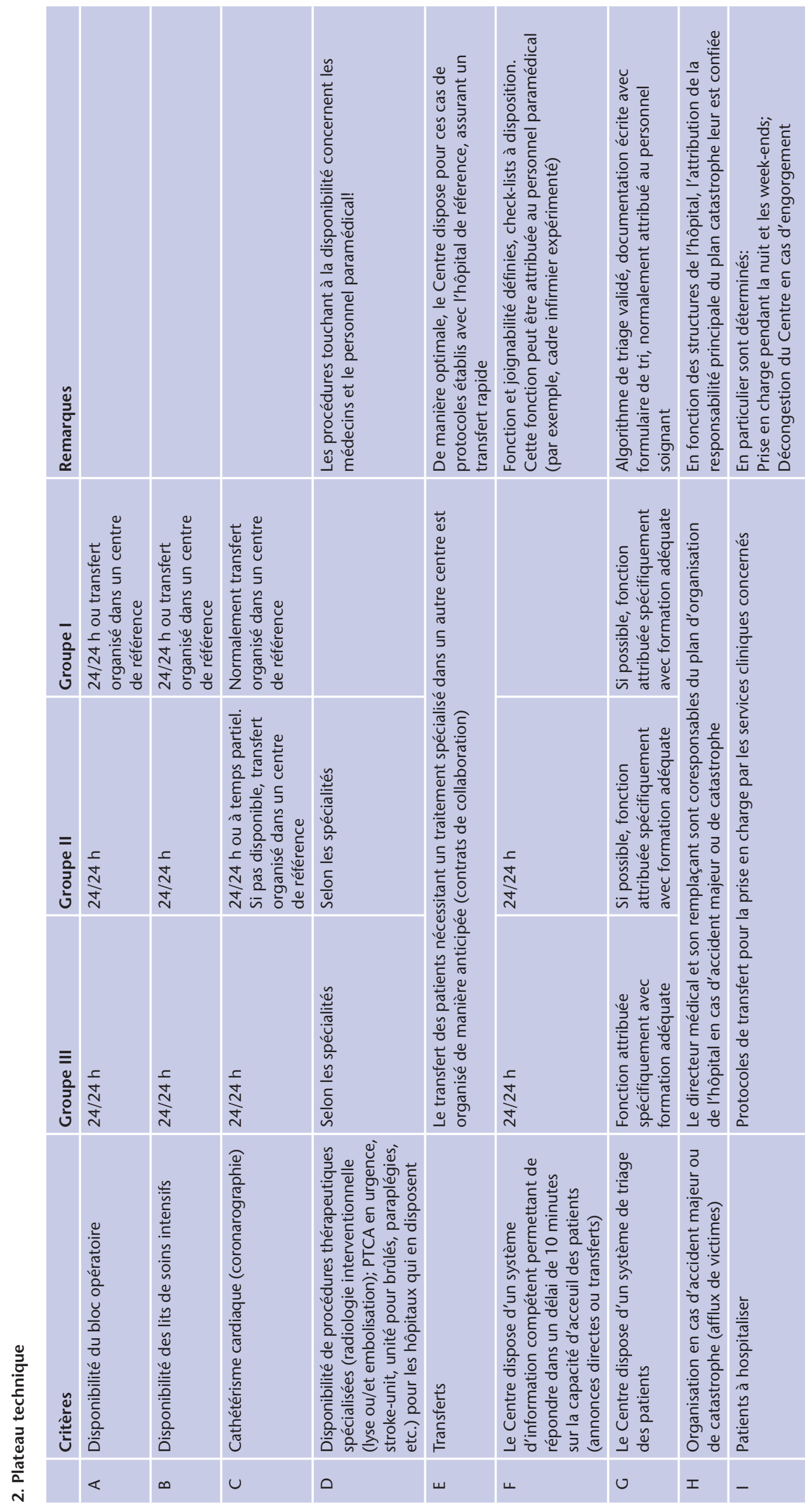




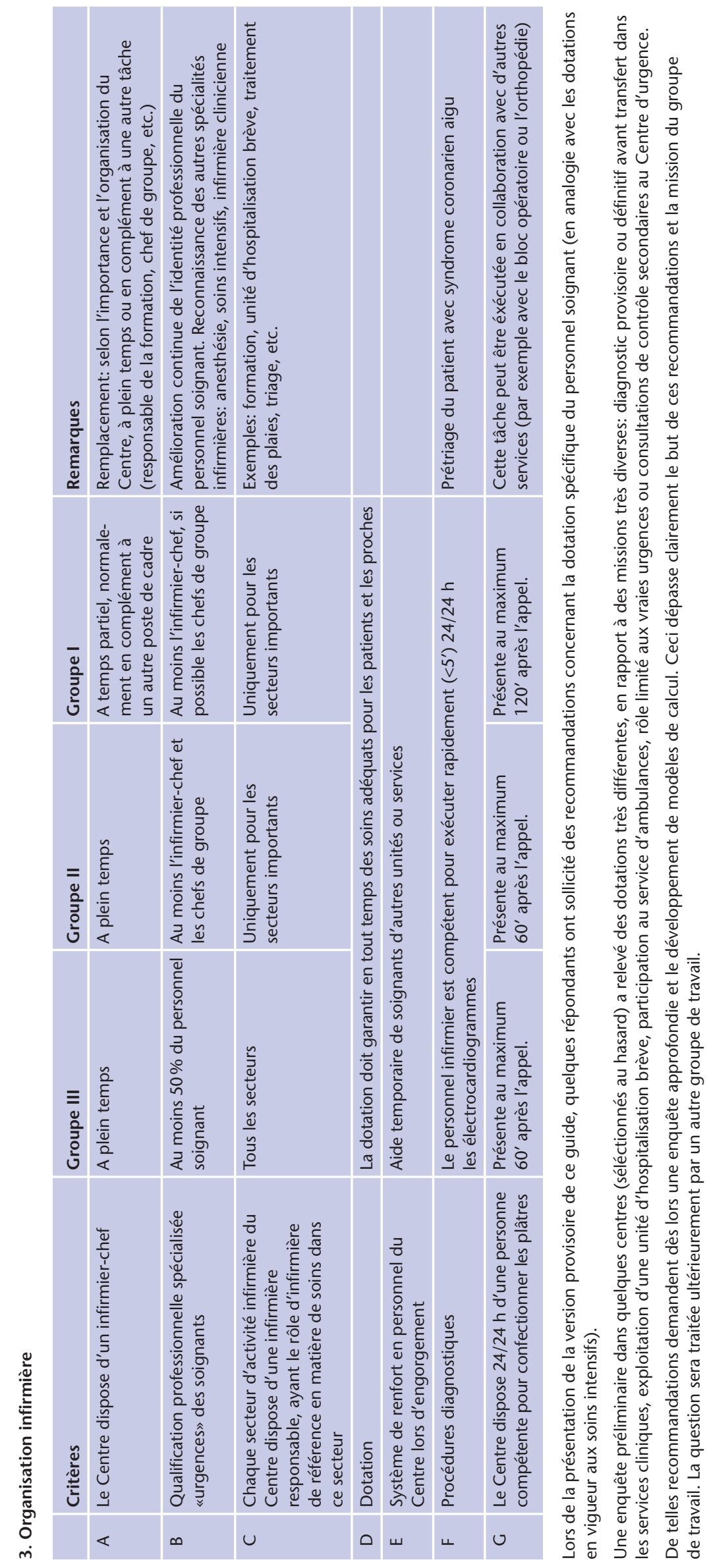




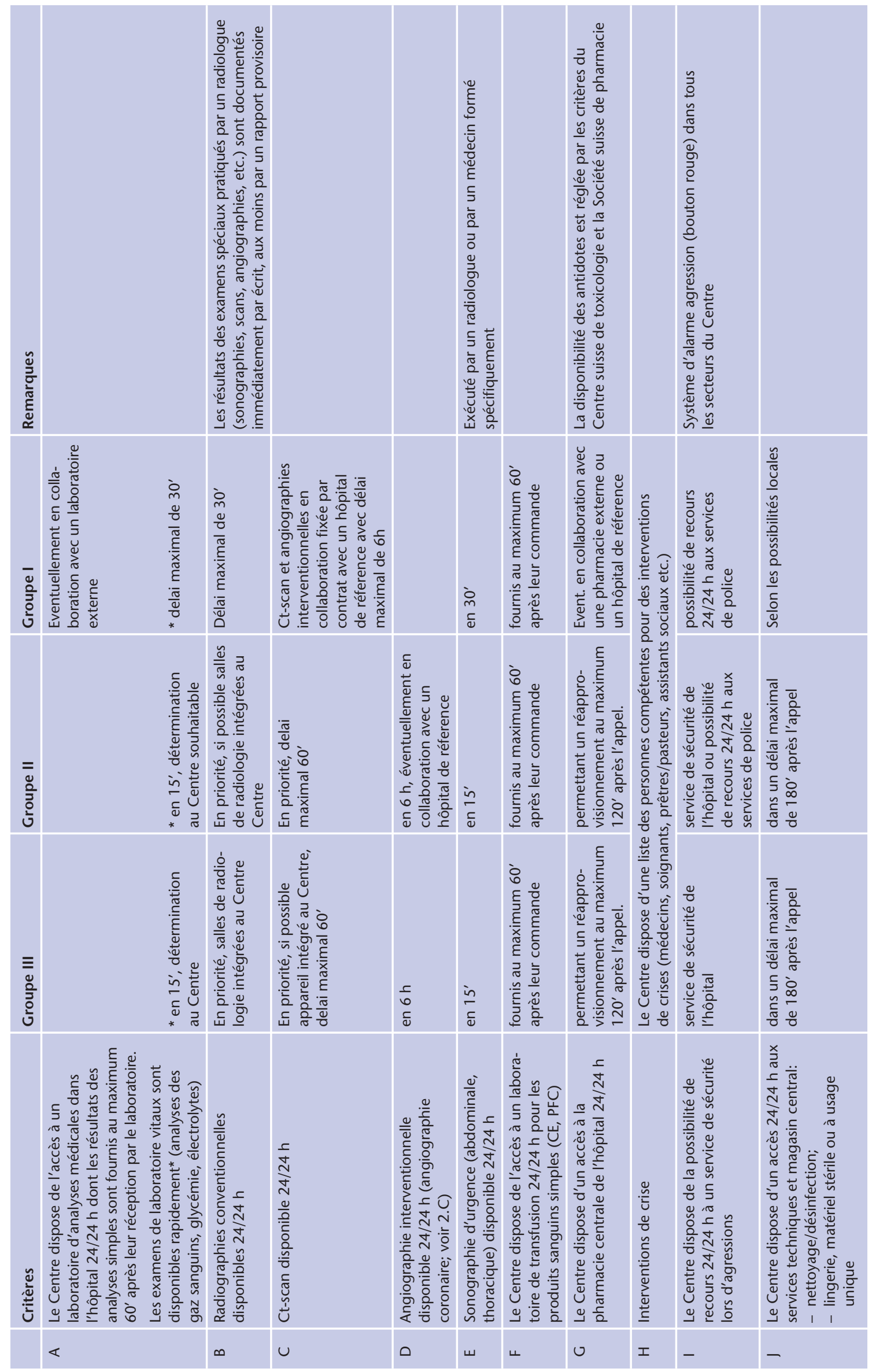




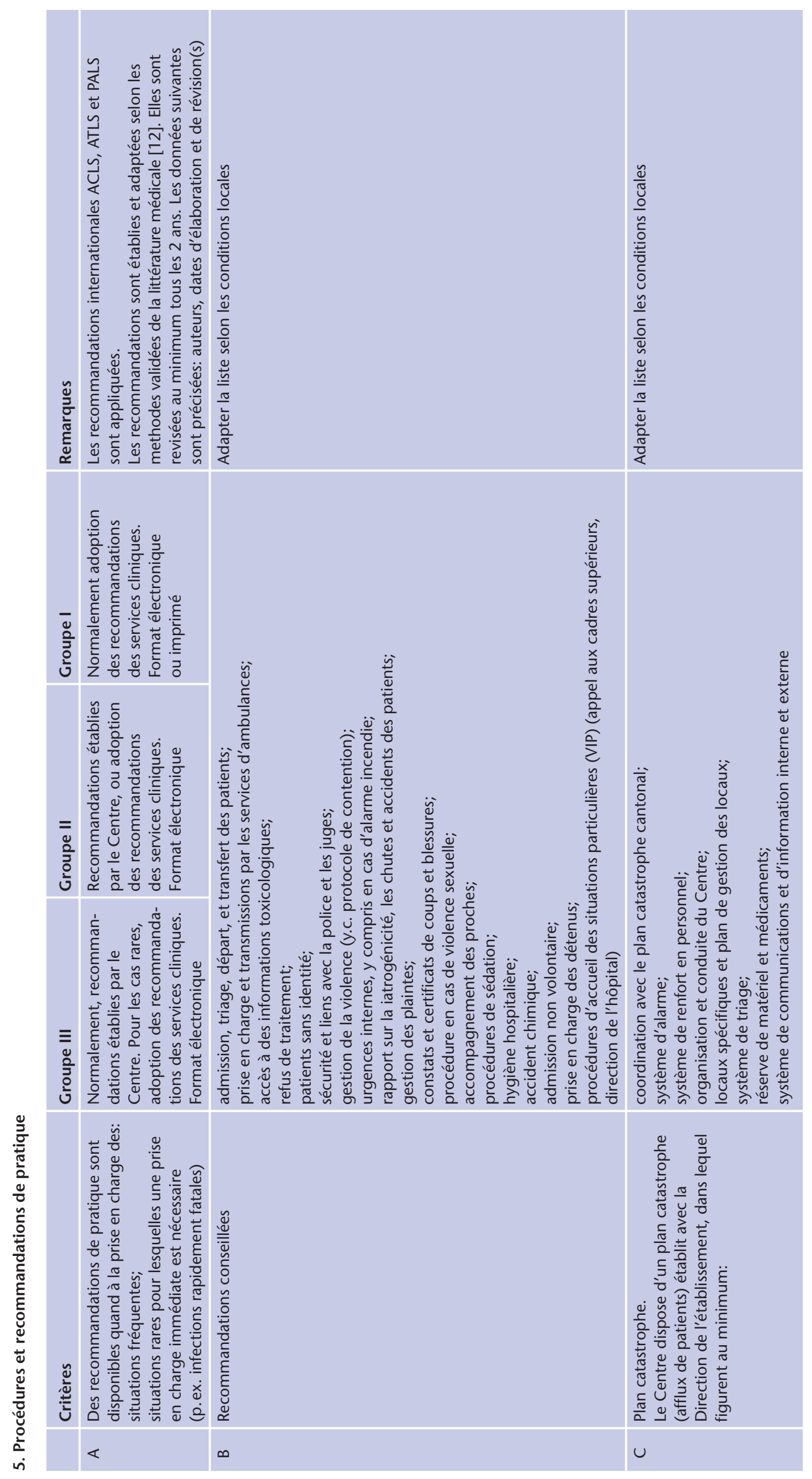




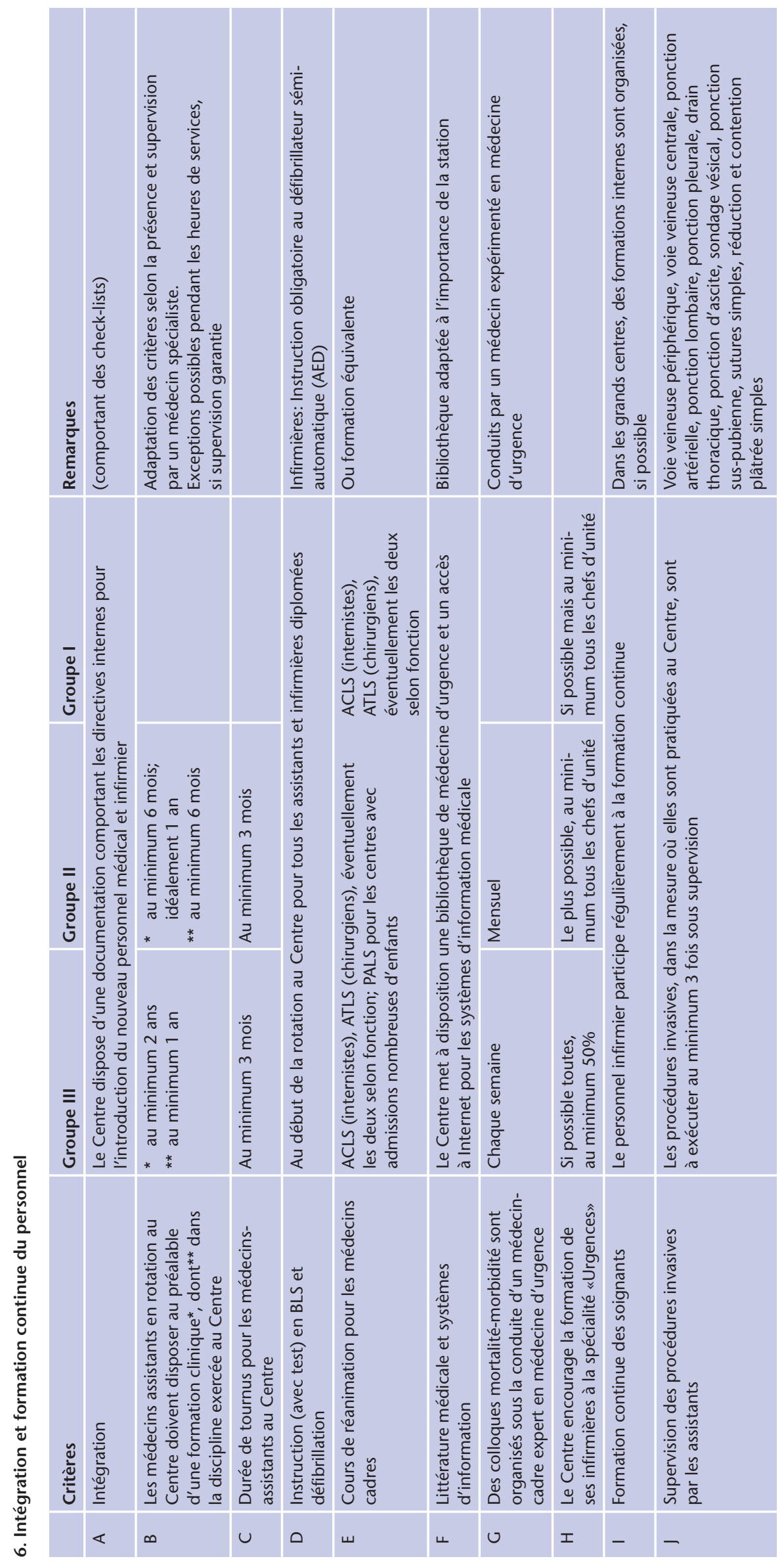




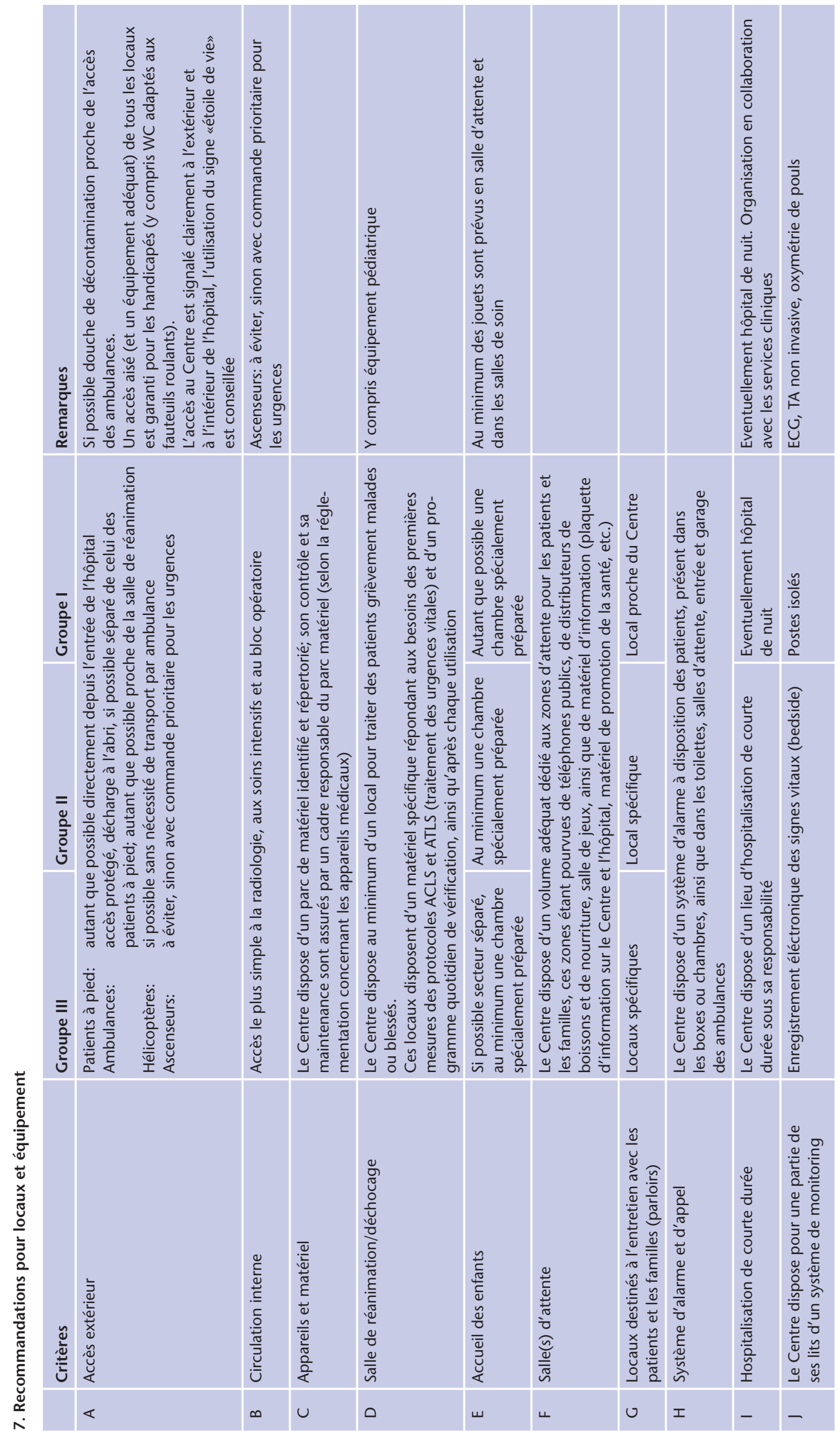



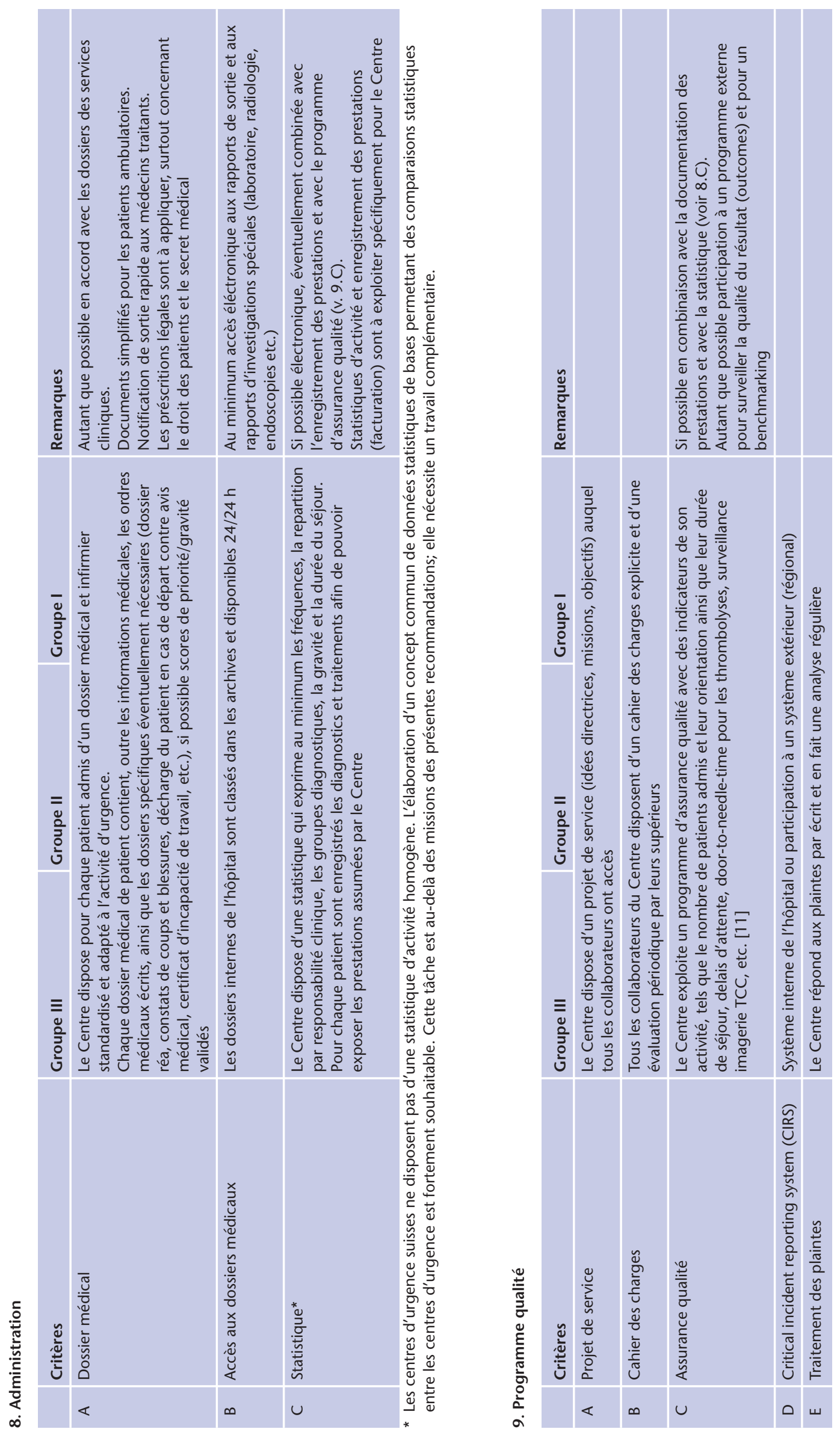


\section{Bibliographie}

1 Irish Society for Quality in Healthcare. Accident and emergency department assessment standards. Dublin, 2000. www.isqh.ie.

2 Commission de médecine d'urgence de la Société de réanimation de langue française (SRLF). Recommandations concernant l'évaluation de l'activité des services d'accueil des urgences (SAU). Réan Urg 1994;3:299-306.

3 Commission d'évaluation de la Société francophone de médecine d'urgence (SFMU). Critères d'évaluation des services d'urgence. Mars 2001. www.sfmu.fr.

4 Commission d'évaluation de la Société francophone de médecine d'urgence (SFMU). Audit d'un service d'urgence; méthode. Mars 1999. www.sfmu.fr.

5 Gerbeaux P, Bourrier P, Chéron G, Fourestié V, Goralski M, Jacquet-Francillon T. Recommandations de la Société francophone de médecine d'urgence concernant la mise en place, la gestion, l'utilisation et l'évaluation des unités d'hospitalisation de courte durée des services d'urgence. JEUR 2001;14:144-52.

6 Rosen RA (ed.). Managing to get it right: the ACEP user's guide to emergency department management. Dallas: American College of Emergency Physicians (ACEP); 1998.
7 Huddy Jon (ed.). Emergency department design; a practical guide to planning for the future. Dallas: American College of Emergency Physicians (ACEP); 2002.

8 Commission des urgences de la Société de réanimation de langue française (SRLF) et Commission d'évaluation de la Société francophone de médecine d'urgence (SFMU). Guide des outils d'évaluation aux urgences. Paris: Arnette \& Blackwell; 1996.

9 American Academy of Pediatrics. Care of children in the emergency department: guidelines for preparedness. Pediatrics 2001;107:777-81.

10 Groupe de travail du Ministère de la santé et des services sociaux du Gouvernement du Québec. Projet Urgence 2000; de paratonnerre à plaque tournante (document de réflexion). Montréal: Ministère de la santé et des services sociaux du Gouvernement du Québec; 1999.

11 Donabedian A. The quality of care; how can it be assessed? JAMA 1988;260:1743-8.

12 Stiell IG, Wells GA. Methodologic standards for the development of clinical decision rules in emergency medicine. Ann Emerg Med 1999;33: 437-47. 